\title{
Improved Prediction Dynamics for Robust MPC
}

This paper was downloaded from TechRxiv (https://www.techrxiv.org).

\section{LICENSE}

CC BY 4.0

SUBMISSION DATE / POSTED DATE

$17-01-2022$ / 24-01-2022

CITATION

Hoai Nam, Nguyen (2022): Improved Prediction Dynamics for Robust MPC. TechRxiv. Preprint. https://doi.org/10.36227/techrxiv.18526274.v1

$\mathrm{DOI}$

10.36227/techrxiv.18526274.v1 


\title{
Improved Prediction Dynamics for Robust MPC
}

\author{
H.-N. Nguyen ${ }^{\dagger}$
}

\begin{abstract}
The objectives of this paper are twofold. The first is to present a particular choice of the parameters of dynamic feedback laws for polytopic uncertain and/or time-varying systems with state and input constraints. We show that it has the same desired property as that of algorithms in [1], [2], i.e., the domain of attraction of the controlled system under a linear/saturated dynamic feedback law is identical to the domain of attraction under any static linear/saturated state feedback law. The second objective is to propose new procedures for robust constrained prediction dynamics based MPC that do not require the assumption of quadratic stability. With respect to other well known techniques, the main advantages of this new approach is the reduced conservativeness.
\end{abstract}

\section{INTRODUCTION}

\section{A. Introduction and Problem Formulation}

Consider the following uncertain and/or time-varying linear discrete-time system

$$
x(k+1)=A(\lambda(k)) x(k)+B(\lambda(k)) u(k)
$$

where $x(k) \in \mathbb{R}^{n}$ is the measured state, $u(k) \in \mathbb{R}^{m}$ is the control input. The matrices $A(\lambda(k)) \in$ $\mathbb{R}^{n \times n}, B(\lambda(k)) \in \mathbb{R}^{n \times m}$ satisfy

$$
\left\{\begin{array}{l}
A(\lambda(k))=\sum_{i=1}^{s} \lambda_{i}(k) A_{i}, B(\lambda(k))=\sum_{i=1}^{s} \lambda_{i}(k) B_{i}, \\
\sum_{i=1}^{s} \lambda_{i}(k)=1, \lambda_{i}(k) \geq 0
\end{array}\right.
$$

where $A_{i} \in \mathbb{R}^{n \times n}, B_{i} \in \mathbb{R}^{n \times m}$ are known matrices. $\lambda(k)=\left[\lambda_{1}(k) \lambda_{2}(k) \ldots \lambda_{s}(k)\right]^{T}$ is a vector of parametric uncertainties such that

$$
\lambda(k) \in \Lambda:\left\{\sum_{i=1}^{s} \lambda_{i}=1, \lambda_{i} \geq 0, \forall i=\overline{1, s}\right\}
$$

$\dagger$ Electronics and Physics Department - Telecom-SudParis, 9 rue Charles Fourier, 91011 Evry Cedex France hoai-nam.nguyen@telecom-sudparis.eu 
It is underlined that $\lambda_{i}$ are unknown and time-varying.

The state $x(k)$ and input $u(k)$ are subject to symmetric linear constraints

$$
\left\{\begin{array}{l}
-1 \leq u_{j} \leq 1, \forall j=\overline{1, m} \\
-1 \leq f_{l} x \leq 1, \forall l=\overline{1, n_{c}}
\end{array}\right.
$$

where $f_{l}$ is the $l$ th row of $F \in \mathbb{R}^{n_{c} \times n}$.

The objective is to design a control law $u(k)=U(x(k))$ such that the controlled system

$$
x(k+1)=A(\lambda(k)) x(k)+B(\lambda(k)) U(x(k))
$$

fulfills the input and state constraints (4) despite the uncertainties. Furthermore, $U(x(k))$ should solve the following min-max problem

$$
\begin{aligned}
& \min _{\{u(k), u(k+1), \ldots,\}} \max _{\lambda(k) \in \Lambda}\{J(k)\} \\
& J(k)=\sum_{t=0}^{\infty}\left(x(k+t)^{T} Q x(k+t)+u(k+t)^{T} R u(k+t)\right)
\end{aligned}
$$

where $x(k+t), u(k+t), t=0,1, \ldots$, are the predicted states and the predicted inputs from time k. $Q \succeq 0, R \succ 0$ are weighting matrices.

In the absence of the constraints (4), it is well known that (5) is a linear quadratic (LQ) regulator problem. The solution is the linear state feedback controller

$$
u(k)=K x(k)
$$

where the gain $K \in \mathbb{R}^{m \times n}$ can be found by solving a semi-definite program (SDP).

In the presence of (4), the problem (5) is intractable due to the need of guaranteeing (2) for the infinite number of constraints. A way to overcome this problem is to employ the following dynamic control law [1], [3], [4]

$$
\left\{\begin{array}{l}
u(k)=K x(k)+N v(k) \\
v(k+1)=\sum_{i=1}^{s} \lambda_{i}(k) M_{i} v(k)
\end{array}\right.
$$

where $v(k) \in \mathbb{R}^{n_{v}}$ is the controller state, $N$ and $M_{i}$ are unknown matrices that will be treated as decision variables. The uncertain parameters $\lambda_{i}$ are the same as in (3). As will be seen later, knowledge of the $\lambda_{i}$ is not required for the implementation of the control law (8).

The method in [1], [3], [4] consists of offline and online stages. In the offline stage, the parameters $N, M_{i}, \forall i=\overline{1, s}$ are optimized in order to maximize the domain of attraction of the closed-loop system (1) under the control law (8). Once $N, M_{i}, \forall i=\overline{1, s}$ were determined, the 
next step is to estimate an upper bound of the cost function $J(k)$. In the online stage, $v(k)$ is optimized by minimizing the obtained upper bound of $J(k)$ at each sampling time. Then the control law $u(k)=K x(k)+N v(k)$ is applied to (1). In [1], to design $N, M_{i}$, and to calculate the upper bound of $J(k)$, common quadratic Lyapunov functions are used. Hence, the result might be conservative in the sense that only a small amount of uncertainty is allowed. This is due to the fact that the same Lyapunov matrix must verify for all vertices of the uncertain domain (2). In addition, to optimize $N, M_{i}, \forall i=\overline{1, s}$, a lifting process in conjunction with a nonlinear parameter transformation technique are employed. Hence, the resulting optimization problem is in high dimension.

Recently in [2], it is noticed that despite an efficient online computation, the full control range of the prediction dynamics based MPC method with linear feedback (8) is rarely exploited. Hence the time to regulate the plant to the origin is often much longer than necessary. To overcome this problem, a new prediction dynamics based MPC method with saturated feedback was proposed [2]. It was shown that by allowing the parameters of the dynamic control law to depend not only on the system uncertainty, but also on the saturated inputs, the control range is fully exploited. Hence, the performance can be significantly improved. However the method in [2] is still based on common quadratic functions in order to optimize the parameters of the saturated dynamic feedback law, to describe the domain of attraction, and to calculate the upper bound of the cost function.

The main contribution of this paper consists of improving the control techniques presented in [1], [2], by proposing new procedures for robust constrained prediction dynamics based MPC. The new methods do not require the quadratic stalizability of the given uncertain system. Compared with the MPC techniques in [1], [2], the main differences are: i) to describe the domain of attraction we do not use a single ellipsoid but the intersection of ellipsoids, each one corresponds to a different vertex of the uncertainty polytope and/or of the input saturations, ii) to calculate the upper bound of the cost function, instead of a time-invariant, a time-varying Lyapunov function is employed. The online computational burden of the new procedures is generally higher than that of [1], [2], but is still in the milisecond range. Another main contribution of the present paper is that we propose a particular choice of the parameters of the linear/saturated dynamic control laws without solving any optimization problem. This choice has the same desired property as that of algorithms in [1], [2], i.e., the domain of attraction of the controlled system under a linear/saturated dynamic feedback law is identical to the domain of attraction under any static 
linear/saturated state feedback law.

It is stressed that there are other robust min-max MPC techniques for solving the problem (1), (4), (5), e.g., [5], [6], [7]. At each sampling time, the control signal is obtained by minimizing the worst case of (5), which is in turn computed by maximizing over the possible cases of uncertainty. Solving these problems can be computationally costly as they are NP-hard.

This paper is organized as follows. Section II is dedicated to the prediction dynamics based MPC method with linear feedback. In Section III, results on the design of the new prediction dynamics control law with saturated feedback are presented. Two simulated examples with comparison to earlier solutions are evaluated in Section IV before drawing the conclusions in Section V.

\section{B. Preliminaries}

Notation: A positive definite (semidefinite) matrix $P$ is denoted by $P \succ 0(P \succeq 0)$. For a given $P \in \mathbb{R}^{n \times n}, P \succ 0, \mathcal{E}(P)$ represents the following ellipsoid

$$
\mathcal{E}(P)=\left\{x \in \mathbb{R}^{n}: x^{T} P^{-1} x \leq 1\right\}
$$

Let $h_{l}$ be the $l$ th row of the matrix $H \in \mathbb{R}^{n_{h} \times n}, \mathcal{L}(H)$ is used to denote the following symmetric polyhedron

$$
\mathcal{L}(H)=\left\{x \in \mathbb{R}^{n}:-1 \leq h_{l} x \leq 1, \forall l=\overline{1, n_{h}}\right\}
$$

For symmetric matrices, the symbol $(*)$ denotes each of its symmetric block. $\mathbf{0}, \mathbf{I}$ are, respectively, the zero matrix and the identity matrix of appropriate dimension.

Consider the following ellipsoid $\mathcal{E}_{x_{1} x_{2}} \subseteq \mathbb{R}^{n_{1}+n_{2}}$

$$
\left[\begin{array}{ll}
x_{1}^{T} & x_{2}^{T}
\end{array}\right]\left[\begin{array}{ll}
P_{11} & P_{12} \\
P_{12}^{T} & P_{22}
\end{array}\right]^{-1}\left[\begin{array}{l}
x_{1} \\
x_{2}
\end{array}\right] \leq 1
$$

Definition 1: (Projection) Given the ellipsoid $\mathcal{E}_{x_{1} x_{2}}$, the orthogonal projection $E_{x_{1}}$ of $\mathcal{E}_{x_{1} x_{2}}$ onto the $x_{1}$-space $\mathbb{R}^{n_{1}}$ is defined as

$$
E_{x_{1}}=\left\{x_{1} \in \mathbb{R}^{n_{1}}: \exists x_{2} \in \mathbb{R}^{n_{2}} \text { such that }\left[\begin{array}{ll}
x_{1}^{T} & x_{2}^{T}
\end{array}\right] \in \mathcal{E}_{x_{1} x_{2}}\right\}
$$

It is well known that $E_{x_{1}}=\mathcal{E}\left(P_{11}\right)$, i.e.,

$$
E_{x_{1}}=\left\{x_{1} \in \mathbb{R}^{n_{1}}: x_{1}^{T} P_{11}^{-1} x_{1} \leq 1\right\}
$$


The cut of the ellipsoid $\mathcal{E}_{x_{1} x_{2}} \subseteq \mathbb{R}^{n_{1}+n_{2}}$ through $x_{2}=\mathbf{0}$ is given as

$$
\left[\begin{array}{ll}
x_{1}^{T} & \mathbf{0}
\end{array}\right]\left[\begin{array}{ll}
P_{11} & P_{12} \\
P_{12}^{T} & P_{22}
\end{array}\right]^{-1}\left[\begin{array}{l}
x_{1} \\
\mathbf{0}
\end{array}\right] \leq 1
$$

It can be shown that $(11)$ is the ellipsoid $\mathcal{E}\left(P_{11}-P_{12} P_{22}^{-1} P_{12}^{T}\right)$, i.e.,

$$
x_{1}^{T}\left(P_{11}-P_{12} P_{22}^{-1} P_{12}^{T}\right)^{-1} x_{1} \leq 1
$$

Definition 2: (Invariance) A set $\Omega$ is said to be robustly invariant for system (1) if $\forall x(k) \in \Omega$, $\exists u(k)=U(x(k))$ such that $x(k+1) \in \Omega$. In addition, if $\Omega \in \mathcal{L}(F)$ and $-1 \leq U(x(k)) \leq 1$, then $\Omega$ is robustly invariant and constraint-admissible.

Lemma 1: [8] For given $P \in \mathbb{R}^{n \times n}, P \succ 0, f_{0} \in \mathbb{R}^{1 \times n}, \mathcal{E}(P) \subseteq \mathcal{L}\left(f_{0}\right)$ if and only if $f_{0} P f_{0}^{T} \leq 1$.

Lemma 2: [9] Given matrices $P, G$ of appropriate dimension, $P \succ 0$. Then,

$$
G^{T} P^{-1} G \succeq G^{T}+G-P
$$

\section{Prediction Dynamics with Linear FeEdBack}

\section{A. Parameters Optimization}

Combining (1), (8), one obtains

$$
z(k+1)=\mathcal{A}(k) z(k)
$$

where

$$
\begin{aligned}
& z(k)=\left[\begin{array}{c}
x(k) \\
v(k)
\end{array}\right], \mathcal{A}(k)=\sum_{i=1}^{s} \lambda_{i}(k) \mathcal{A}_{i}, \\
& \mathcal{A}_{i}=\left[\begin{array}{cc}
A_{i}+B_{i} K & B_{i} N \\
\mathbf{0}_{n_{v} \times n} & M_{i}
\end{array}\right], \forall i=\overline{1, s}
\end{aligned}
$$

Define

$$
\left\{\begin{array}{l}
\mathbf{K}=\left[\begin{array}{ll}
K & N
\end{array}\right] \\
\mathbf{F}=\left[\begin{array}{ll}
F & \mathbf{0}_{n_{c} \times n}
\end{array}\right]
\end{array}\right.
$$


It is well known [1], [10] that invariance and constraint admissibility of an ellipsoid $\mathcal{E}(P)=$ $\left\{z \in \mathbb{R}^{n+n_{v}}: z^{T} P^{-1} z \leq 1\right\}$ for the closed-loop system (14), and for the constraints (4) are equivalent to

$$
\begin{aligned}
& {\left[\begin{array}{cc}
P & \mathcal{A}_{i} P \\
P \mathcal{A}_{i}^{T} & P
\end{array}\right] \succeq 0, \forall i=\overline{1, s}} \\
& 1-\mathbf{K}_{j} P \mathbf{K}_{j}^{T} \geq 0, \forall j=\overline{1, m} \\
& 1-\mathbf{F}_{l} P \mathbf{F}_{l}^{T} \geq 0, \forall l=\overline{1, n_{c}}
\end{aligned}
$$

where $\mathbf{K}_{j}, \mathbf{F}_{l}$ are, respectively, the $j$ th and $l$ th row of $\mathbf{K}$, and $\mathbf{F}$.

In the control strategy [1], $N, M_{i}, i=\overline{1, s}$ are optimized in order to maximize the domain of attraction. This is done by maximizing the volume of the projection $E_{x}$ of $\mathcal{E}(P)$ onto the $x$-subspace. The optimization problem is non-convex because (16), (17) are bilinear matrix inequalities (BMI) in $P, N, M_{i}$. As shown in [1], the problem can be convexified through the use of a nonlinear parameter transformation. In addition, two other main results are obtained in $[1]$.

(i) In terms of the size of the domain of attraction, there is no advantage to be gained by using $n_{v}>n$.

(ii) With $n_{v}=n$, the maximum volume of $E_{x}$ under the control law (8) can be as large as the volume of the largest invariant and constraint-admissible ellipsoid obtained under any robustly stabilizing static state feedback law, $L \in \mathbb{R}^{m \times n}$

$$
u(k)=L x(k)
$$

The first aim of this paper is to present a particular choice of $N, M_{i}$ such that the two results (i), (ii) in [1] hold for the given matrix gain $L$. This is done without solving any optimization problem. For this purpose, define $\Omega_{L}$ as a robustly invariant and constraint-admissible set for system (1) and for constraints (4) under the controller (19). Note that $\Omega_{L}$ can be any kind of set, and is not needed to be an ellipsoid. Consider the following choice for $N, M_{i}, \forall i=\overline{1, s}$

$$
N=L-K, M_{i}=A_{i}+B_{i} L
$$

Define $\Omega_{z}$ as an invariant and constraint-admissible set for (14), (4), (20). Define also $\Omega_{x}$ as the projection of $\Omega_{z}$ onto the $x$-subspace.

Theorem 1: For $N, M_{i}$ given in (20), $\Omega_{z}$ can be optimized in such a way that $\Omega_{L} \subseteq \Omega_{x}$. 
Proof: Since $\Omega_{L}$ is a robustly invariant and constraint-admissible set for (1), (4) under (19), it follows that $\forall x(k) \in \Omega_{L}$

$$
x(k+1)=\sum_{i=1}^{s} \lambda_{i}(k)\left(A_{i}+B_{i} L\right) x(k) \in \Omega_{L}
$$

and

$$
\left\{\begin{array}{l}
-1 \leq L_{j} x(k) \leq 1, \forall j=\overline{1, m} \\
-1 \leq f_{l} x(k) \leq 1, \forall l=\overline{1, n_{c}}
\end{array}\right.
$$

where $L_{j}$ is the $j$ th row of $L$. Using (20), rewrite the closed-loop system (14) as

$$
\left[\begin{array}{c}
x(k+1) \\
v(k+1)
\end{array}\right]=\sum_{i=1}^{s} \lambda_{i}(k)\left[\begin{array}{cc}
A_{i}+B_{i} K & B_{i}(L-K) \\
\mathbf{0}_{n \times n} & A_{i}+B_{i} L
\end{array}\right]\left[\begin{array}{l}
x(k) \\
v(k)
\end{array}\right]
$$

Using (8), (20), the constraints (4) become

$$
\left\{\begin{array}{l}
-1 \leq K_{j} x(k)+\left(K_{j}-L_{j}\right) v(k) \leq 1, \forall j=\overline{1, m} \\
-1 \leq f_{l} x(k) \leq 1, \forall l=\overline{1, n_{c}}
\end{array}\right.
$$

where $K_{j}$ is the $j$ th row of $K . \forall z(k)$ such that $x(k)=v(k)$, one has

$$
\begin{aligned}
x(k+1) & =\sum_{i=1}^{s} \lambda_{i}(k)\left(\left(A_{i}+B_{i} K\right) x(k)+B_{i}(L-K) x(k)\right) \\
& =\sum_{i=1}^{s} \lambda_{i}(k)\left(A_{i}+B_{i} L\right) x(k) \\
& =\sum_{i=1}^{s} \lambda_{i}(k)\left(A_{i}+B_{i} L\right) v(k)=v(k+1)
\end{aligned}
$$

Hence (23) consists of two identically decoupled systems. The constraints (24) become (22) for $x(k)$. It follows that $\Omega_{L} \subseteq \Omega_{x}$.

Note that with the choice (20), the information of the system dynamics (1) are heavily exploited. In the rest of the paper, (20) will be used for the linear dynamic control law (8).

\section{B. Maximal Stabilizable Set}

In this section, we show how to calculate a robustly invariant and constraint-admissible set of the closed-loop system (14) with the constraints (4).

Two classes of invariant sets are generally considered for system (14). The first one is ellipsoidal invariant sets, which correspond to quadratic Lyapunov functions, and are the most commonly used. Their popularity is due to computational efficiency via the use of linear matrix inequality (LMI) formulation, and the complexity is fixed with respect to the dimension of the state space. However, it is well known [11] that there exist asymptotically stable uncertain systems 
which do not admit quadratic Lyapunov functions. The second class is polyhedral invariant sets. In general, polyhedral invariant sets are preferred to the ellipsoidal ones, as they form a universal class of Lyapunov functions [11]. However, constructing a polyhedral invariant set is generally harder than the computation of an ellipsoidal one, especially for uncertain systems and/or for the large state dimension [11], [10].

In recent years, other types of non-quadratic Lyapunov functions are considered for discretetime systems with constraints, e. g., [12], [13]. The Lyapunov functions in these works pertain to or are composed from several quadratic functions. However they lead to optimization problems with BMI constraints, which are non-convex.

In this paper, we follow the idea in [13], i.e., we use the intersection of ellipsoids to characterize the domain of attraction. The main contribution with respect to [13] is that the conditions are expressed as LMI constraints, which are convex.

Theorem 2: If there exist matrices $P_{i} \in \mathbb{R}^{2 n \times 2 n}, P_{i} \succ 0, \forall i=\overline{1, s}$ such that the following conditions hold

$$
\begin{gathered}
{\left[\begin{array}{cc}
P_{i_{1}} & \mathcal{A}_{i} P_{i} \\
P_{i} \mathcal{A}_{i}^{T} & P_{i}
\end{array}\right] \succeq 0, \forall i, \forall i_{1}=\overline{1, s}} \\
1-\mathbf{K}_{j} P_{i_{2}} \mathbf{K}_{j}^{T} \geq 0, \exists i_{2} \in \overline{1, s}, \forall j=\overline{1, m} \\
1-\mathbf{F}_{l} P_{i_{3}} \mathbf{F}_{l}^{T} \geq 0, \exists i_{3} \in \overline{1, s}, \forall l=\overline{1, n_{c}}
\end{gathered}
$$

then the intersection of ellipsoids $\bigcap_{i=1}^{s} \mathcal{E}\left(P_{i}\right)$ is robustly invariant and constraint-admissible for (14), (4).

Proof: For robust invariance, one needs to show that $z(k+1) \in \bigcap_{i=1}^{s} \mathcal{E}\left(P_{i}\right), \forall z(k) \in \bigcap_{i=1}^{s} \mathcal{E}\left(P_{i}\right)$. Pre- and post-multiplication of (26) by

$$
\left[\begin{array}{cc}
P_{i_{1}}^{-1} & \mathbf{0}_{n \times n} \\
\mathbf{0}_{n \times n} & P_{i}^{-1}
\end{array}\right]
$$

one obtains

$$
\left[\begin{array}{cc}
P_{i_{1}}^{-1} & P_{i_{1}}^{-1} \mathcal{A}_{i} \\
\mathcal{A}_{i}^{T} P_{i_{1}}^{-1} & P_{i}^{-1}
\end{array}\right] \succeq 0, \forall i, \forall i_{1}=\overline{1, s}
$$

For each $i_{1}$, multiply the corresponding $i=\overline{1, s}$ by $\lambda_{i}(k)$, and sum to get

$$
\left[\begin{array}{cc}
P_{i_{1}}^{-1} & P_{i_{1}}^{-1}\left(\sum_{i=1}^{s} \lambda_{i}(k) \mathcal{A}_{i}\right) \\
\left(\sum_{i=1}^{s} \lambda_{i}(k) \mathcal{A}_{i}\right)^{T} P_{i_{1}}^{-1} & \left(\sum_{i=1}^{s} \lambda_{i}(k) P_{i}^{-1}\right)
\end{array}\right] \succeq 0, \forall i_{1}=\overline{1, s}
$$


Using Schur complement, one obtains

$$
\left(\sum_{i=1}^{s} \lambda_{i}(k) P_{i}^{-1}\right)-\left(\sum_{i=1}^{s} \lambda_{i}(k) \mathcal{A}_{i}\right)^{T} P_{i_{1}}^{-1}\left(\sum_{i=1}^{s} \lambda_{i}(k) \mathcal{A}_{i}\right) \succeq 0
$$

thus, $\forall i_{1}=\overline{1, s}$

$$
z(k)^{T}\left(\sum_{i=1}^{s} \lambda_{i}(k) P_{i}^{-1}\right) z(k)-z(k)^{T}\left(\sum_{i=1}^{s} \lambda_{i}(k) \mathcal{A}_{i}\right)^{T} P_{i_{1}}^{-1}\left(\sum_{i=1}^{s} \lambda_{i}(k) \mathcal{A}_{i}\right) z(k) \geq 0
$$

Note that $z(k+1)=\left(\sum_{i=1}^{s} \lambda_{i}(k) \mathcal{A}_{i}\right) z(k)$. Hence, $\forall i_{1}=\overline{1, s}$

$$
z(k)^{T}\left(\sum_{i=1}^{s} \lambda_{i}(k) P_{i}^{-1}\right) z(k)-z(k+1)^{T} P_{i_{1}}^{-1} z(k+1) \geq 0
$$

One has $z(k) \in \mathcal{E}\left(P_{i}\right), \forall i=\overline{1, s}, \forall z(k) \in \bigcap_{i=1}^{s} \mathcal{E}\left(P_{i}\right)$, or equivalently

$$
z(k)^{T} P_{i}^{-1} z(k) \leq 1, \forall i=\overline{1, s}
$$

thus

$$
\begin{aligned}
z(k)^{T}\left(\sum_{i=1}^{s} \lambda_{i}(k) P_{i}^{-1}\right) z(k) & =\sum_{i=1}^{s} \lambda_{i}(k) z(k)^{T} P_{i}^{-1} z(k) \\
& \leq \sum_{i=1}^{s} \lambda_{1}(k)=1
\end{aligned}
$$

Combining with (29), one gets

$$
z(k+1)^{T} P_{i_{1}}^{-1} z(k+1) \leq 1, \forall i_{1}=\overline{1, s}
$$

Hence $z(k+1) \in \mathcal{E}\left(P_{i_{1}}\right), \forall i_{1}=\overline{1, s}$, or equivalently, $z(k+1) \in \bigcap_{i_{1}=1}^{s} \mathcal{E}\left(P_{i_{1}}\right)$.

It remains to prove the constraint admissibility (4). Since $\bigcap_{i=1}^{s} \mathcal{E}\left(P_{i}\right)$ is robustly invariant, it suffices to guarantee

$$
\left\{\begin{array}{l}
\bigcap_{i=1}^{s} \mathcal{E}\left(P_{i}\right) \subseteq \mathcal{L}\left(\mathbf{K}_{j}\right), \forall j=\overline{1, m} \\
\bigcap_{i=1}^{s} \mathcal{E}\left(P_{i}\right) \subseteq \mathcal{L}\left(\mathbf{F}_{l}\right), \forall l=\overline{1, n_{c}}
\end{array}\right.
$$

For each $j \in \overline{1, m}$, if there exists $i_{2}$ such that $\mathcal{E}\left(P_{i_{2}}\right) \subseteq \mathcal{L}\left(\mathbf{K}_{j}\right)$, then

$$
\bigcap_{i=1}^{s} \mathcal{E}\left(P_{i}\right) \subseteq \mathcal{E}\left(P_{i_{2}}\right) \subseteq \mathcal{L}\left(\mathbf{K}_{j}\right)
$$

Using Lemma 1 , condition $\mathcal{E}\left(P_{i_{2}}\right) \subseteq \mathcal{L}\left(\mathbf{K}_{j}\right)$ is equivalent to

$$
1-\mathbf{K}_{j} P_{i_{2}} \mathbf{K}_{j}^{T} \geq 0
$$


This condition is (27). Similarly for the state constraints, for each $l \in \overline{1, n_{c}}$ if there exists $i_{3}$ such that $\mathcal{E}\left(P_{i_{3}}\right) \subseteq \mathcal{L}\left(\mathbf{F}_{l}\right)$, then

$$
\bigcap_{i=1}^{s} \mathcal{E}\left(P_{i}\right) \subseteq \mathcal{E}\left(P_{i_{3}}\right) \subseteq \mathcal{L}\left(\mathbf{F}_{l}\right)
$$

Using Lemma 1 , one can rewrite $\mathcal{E}\left(P_{i_{3}}\right) \subseteq \mathcal{L}\left(\mathbf{F}_{l}\right)$ as

$$
1-\mathbf{F}_{l} P_{i_{3}} \mathbf{F}_{l}^{T} \geq 0
$$

One obtains (28).

Remark 1: In Theorem 2, if we set $P_{i}=P, \forall i=\overline{1, s}$, then conditions (26), (27), (28) boil down to (16), (17), (18). This implies that Theorem 2 is less conservative than the one that employs a single ellipsoid.

Remark 2: Conditions (27) and (28) should be read as follows: for each $j \in \overline{1, m}$ and each $l \in \overline{1, n_{c}}$, there exists $i_{2} \in \overline{1, s}$ and $i_{3} \in \overline{1, s}$ such that $1-\mathbf{K}_{j} P_{i_{2}} \mathbf{K}_{j}^{T} \geq 0$ and $1-\mathbf{F}_{l} P_{i_{3}} \mathbf{F}_{l}^{T} \geq 0$. For example, consider the case $s=m=2$. Then, the input constraints are satisfied if and only if one of the following four conditions holds
(a) $\left\{\begin{array}{c}1-\mathbf{K}_{1} P_{1} \mathbf{K}_{1}^{T} \geq 0, \\ 1-\mathbf{K}_{2} P_{1} \mathbf{K}_{2}^{T} \geq 0\end{array}\right.$
(b) $\left\{\begin{array}{l}1-\mathbf{K}_{1} P_{1} \mathbf{K}_{1}^{T} \geq 0, \\ 1-\mathbf{K}_{2} P_{2} \mathbf{K}_{2}^{T} \geq 0\end{array}\right.$
(c) $\left\{\begin{array}{l}1-\mathbf{K}_{1} P_{2} \mathbf{K}_{1}^{T} \geq 0, \\ 1-\mathbf{K}_{2} P_{1} \mathbf{K}_{2}^{T} \geq 0\end{array}\right.$
(d) $\left\{\begin{array}{l}1-\mathbf{K}_{1} P_{2} \mathbf{K}_{1}^{T} \geq 0, \\ 1-\mathbf{K}_{2} P_{2} \mathbf{K}_{2}^{T} \geq 0\end{array}\right.$

Decompose matrices $P_{i}, i=\overline{1, s}$ as

$$
P_{i}=\left[\begin{array}{cc}
P_{i, 11} & P_{i, 12} \\
P_{i, 21} & P_{i, 22}
\end{array}\right]
$$

where $P_{i, 11}, P_{i, 12}, P_{i, 21}, P_{i 22} \in \mathbb{R}^{n \times n}, P_{i, 11}=P_{i, 11}^{T}, P_{i, 22}=P_{i, 22}^{T}, P_{i, 12}=P_{i, 21}^{T}$.

The projection of $\bigcap_{i=1}^{s} \mathcal{E}\left(P_{i}\right)$ onto the $x$-subspace is the domain of attraction for the control law (8), (20). Using (10), this set is proportional to $\bigcap_{i=1}^{s} \mathcal{E}\left(P_{i, 11}\right)$ and should be maximized. On the other hand, using (12), the intersection of $\bigcap_{i=1}^{s} \mathcal{E}\left(P_{i}\right)$ and the plane $v=\mathbf{0}_{n \times 1}$ is given as $\bigcap_{i=1}^{s} \mathcal{E}\left(P_{i, 11}-P_{i, 12} P_{i, 22}^{-1} P_{i, 21}\right)$. In this set, the control law (8), (20) becomes $u(k)=K x(k)$, which is the optimal unconstrained LQ controller. $\bigcap_{i=1}^{s} \mathcal{E}\left(P_{i, 11}-P_{i, 12} P_{i, 22}^{-1} P_{i, 21}\right)$ should also be maximized. In the literature, e.g., [1], the largeness of the set is generally measured by its volume. Here following the idea in [8], we take the shape of a set into consideration. 
Let $X_{r} \subset \mathbb{R}^{n}$ be a prescribed compact convex set. Define, for a set $X_{c} \subset \mathbb{R}^{n}$

$$
\sigma_{X_{r}}\left(X_{c}\right)=\min _{\sigma}\left\{\sigma>0: \frac{1}{\sigma} X_{r} \subseteq X_{c}\right\}
$$

If $\sigma \leq 1$ then $X_{r} \subseteq X_{c}$. The reference set $X_{r}$ can be chosen according to the available information on the initial conditions. In general, ellipsoids and polytopes are considered for $X_{r}$.

Define $X_{r i}, Y_{r i}$ as reference sets for $\mathcal{E}\left(P_{i}\right)$, and for $\mathcal{E}\left(P_{i, 11}-P_{i, 12} P_{i, 22}^{-1} P_{i, 21}\right), i=\overline{1, s}$, respectively. Note that $X_{r i}, Y_{r i}$ might be different for different $i=\overline{1, s}$.

The problem of maximizing the size of $\bigcap_{i=1}^{s} \mathcal{E}\left(P_{i, 11}\right)$ and $\bigcap_{i=1}^{s} \mathcal{E}\left(P_{i, 11}-P_{i, 12} P_{i, 22}^{-1} P_{i, 21}\right)$ with respect to shape reference sets can be formulated as

$$
\begin{aligned}
& \min _{\sigma_{i}, \mu_{i}, P_{i}}\left\{\sum_{i=1}^{s} \sigma_{i}+\gamma \sum_{i=1}^{s} \mu_{i}\right\} \\
& \text { s.t. }\left\{\begin{array}{l}
(a) \frac{1}{\sigma_{i}} X_{r i} \subseteq \mathcal{E}\left(P_{i, 11}\right), \forall i=\overline{1, s} \\
\text { (b) } \frac{1}{\mu_{i}} Y_{r i} \subseteq \mathcal{E}\left(P_{i, 11}-P_{i, 12} P_{i, 22}^{-1} P_{i, 21}\right), \forall i=\overline{1, s} \\
\text { (c) Conditions }(26),(27),(28)
\end{array}\right.
\end{aligned}
$$

where $\gamma \geq 0$ is a weighting factor.

For simplicity, we only consider the case where $X_{r i}, Y_{r i}$ are ellipsoids. With a slight abuse of notation, $X_{r i}, Y_{r i}$ are used to denote the set as well as the matrices of $\mathcal{E}\left(X_{r i}\right)$, $\mathcal{E}\left(Y_{r i}\right)$, i.e.,

$$
\left\{\begin{array}{l}
X_{r i}=\left\{x \in \mathbb{R}^{n}: x^{T} X_{r i}^{-1} x \leq 1\right\} \\
Y_{r i}=\left\{x \in \mathbb{R}^{n}: x^{T} Y_{r i}^{-1} x \leq 1\right\}
\end{array}\right.
$$

Condition (a) is equivalent to

$$
\frac{1}{\sigma_{i}} X_{r i}^{-1} \succeq P_{i, 11}^{-1}
$$

thus

$$
P_{i, 11}-\sigma_{i} X_{r i} \succeq 0
$$

Condition (b) can be written as

$$
\mu_{i} Y_{r i} \preceq P_{i, 11}-P_{i, 12} P_{i, 22}^{-1} P_{i, 21}
$$

Using Schur complement, one gets

$$
\left[\begin{array}{cc}
P_{i, 11}-\mu_{i} Y_{r i} & P_{i, 12} \\
P_{i, 21} & P_{i, 22}
\end{array}\right] \succeq 0, \forall i=\overline{1, s}
$$

Using (32), (33), the optimization problem (31) can be rewritten as

$$
\min _{\sigma_{i}, \mu_{i}, P_{i}}\left\{\sum_{i=1}^{s} \sigma_{i}+\gamma \sum_{i=1}^{s} \mu_{i}\right\}
$$

s.t. $(26),(27),(28),(32),(33)$ 
(34) is a convex SDP problem. It can be solved efficiently using free available LMI parser such as CVX [14], or Yalmip [15].

\section{Min-max Cost and Overall MPC algorithm}

In this section, a way to compute an upper bound of the cost $J(k)$ is presented. Then the new control scheme is introduced. Consider the following time-varying function

$$
V(k, z(k))=z(k)^{T}\left(\sum_{i=1}^{s} \lambda_{i}(k) \Xi_{i}\right) z(k)
$$

where $\Xi_{i} \in \mathbb{R}^{2 n \times 2 n}, \Xi_{i} \succeq 0, i=\overline{1, s}$ are chosen to satisfy, $\forall t=0,1 \ldots$,

$$
\begin{aligned}
& x(k+t)^{T} Q x(k+t)+u(k+t)^{T} R u(k+t) \leq \\
& \quad \leq V(k+t, z(k+t))-V(k+t+1, z(k+t+1))
\end{aligned}
$$

We will provide a way to compute $\Xi_{i}, i=\overline{1, s}$. For the moment, let us assume that $\Xi_{i}$ are known. Since $K, L$ are robustly stabilizing control laws and $\mathcal{A}(k)$ is an upper triangular matrix, it follows that system (14) is robustly asymptotically stable for states near the origin. Hence $\lim _{t \rightarrow \infty} z(k+t)=0$, or $\lim _{t \rightarrow \infty} V(k+t, z(k+t))=0$. Summing (36) from $t=0$ to $t=\infty$, one gets

$$
\sum_{t=0}^{\infty}\left(x(k+t)^{T} Q x(k+t)+u(k+t)^{T} R u(k+t)\right) \leq V(k, z(k))
$$

or equivalently $J(k) \leq V(k, z(k))$. Hence $V(k, z(k))$ provides an upper bound of $J(k)$.

The following theorem concerns the existence of $\Xi_{i}$ in (36)

Theorem 3: There exist $\Xi_{i}, \forall i=\overline{1, s}$ satisfying (36) if and only if the following conditions hold

$$
\left[\begin{array}{cc}
\Xi_{i}-\mathbf{Q}-\mathbf{R} & \mathcal{A}_{i}^{T} \Xi_{i_{1}} \\
\Xi_{i_{1}} \mathcal{A}_{i} & \Xi_{i_{1}}
\end{array}\right] \succeq 0, \forall i, \forall i_{1}=\overline{1, s}
$$

where

$$
\mathbf{Q}=\left[\begin{array}{cc}
Q & \mathbf{0}_{n \times n} \\
\mathbf{0}_{n \times n} & \mathbf{0}_{n \times n}
\end{array}\right], \mathbf{R}=\mathbf{K}^{T} R \mathbf{K}
$$

Proof: Since $t$ is arbitrary, it suffices to verify (36) for $t=0$. Using (36) and the facts that $x(k)=\left[\begin{array}{ll}\mathbf{I}_{n} & \mathbf{0}_{n}\end{array}\right] z(k)$ and $u(k)=\mathbf{K} z(k)$, one obtains

$$
\left(\sum_{i=1}^{s} \lambda_{i}(k) \Xi_{i}\right)-\left(\sum_{i=1}^{s} \lambda_{i}(k) \mathcal{A}_{i}\right)^{T}\left(\sum_{i_{1}=1}^{s} \lambda_{i_{1}}(k+1) \Xi_{i_{1}}\right)\left(\sum_{i=1}^{s} \lambda_{i}(k) \mathcal{A}_{i}\right) \succeq \mathbf{Q}+\mathbf{R}
$$


Condition (39) holds if and only if, $\forall i_{1}=\overline{1, s}$

$$
\left(\sum_{i=1}^{s} \lambda_{i}(k) \Xi_{i}\right)-\left(\sum_{i=1}^{s} \lambda_{i}(k) \mathcal{A}_{i}\right)^{T} \Xi_{i_{1}}\left(\sum_{i=1}^{s} \lambda_{i}(k) \mathcal{A}_{i}\right) \succeq \mathbf{Q}+\mathbf{R}
$$

Using Schur complement, one obtains, $\forall i_{1}=\overline{1, s}$

$$
\left[\begin{array}{cc}
\left(\sum_{i=1}^{s} \lambda_{i}(k) \Xi_{i}-\mathbf{Q}-\mathbf{R}\right) & \left(\sum_{i=1}^{s} \lambda_{i}(k) \mathcal{A}_{i}\right)^{T} \Xi_{i_{1}} \\
\Xi_{i_{1}}\left(\sum_{i=1}^{s} \lambda_{i}(k) \mathcal{A}_{i}\right) & \Xi_{i_{1}}
\end{array}\right] \succeq 0
$$

or equivalently, $\forall i, \forall i_{1}=\overline{1, s}$

$$
\left[\begin{array}{cc}
\Xi_{i}-\mathbf{Q}-\mathbf{R} & \mathcal{A}_{i}^{T} \Xi_{i_{1}} \\
\Xi_{i_{1}} \mathcal{A}_{i} & \Xi_{i_{1}}
\end{array}\right] \succeq 0
$$

The proof is complete.

Since $\mathcal{A}_{i}$ are upper triangular matrices, there exist $\Xi_{i} \in \mathbb{R}^{2 n \times 2 n}$ satisfying (37), if and only if there exist $\Xi_{i}$ in the following diagonal form

$$
\Xi_{i}=\left[\begin{array}{cc}
\Gamma_{i} & \mathbf{0}_{n \times n} \\
\mathbf{0}_{n \times n} & \Phi_{i}
\end{array}\right], i=\overline{1, s}
$$

where $\Gamma_{i} \in \mathbb{R}^{n \times n}, \Phi_{i} \in \mathbb{R}^{n \times n}, \Gamma_{i} \succeq 0, \Phi_{i} \succeq 0, \forall i=\overline{1, s}$.

$\Xi_{i}$ are used to provide the upper bound of the cost function $J(k)$. The optimal $\Xi_{i}$ can be found by solving the following SDP problem

$$
\left\{\begin{array}{l}
\min _{\Gamma_{i}, \Phi_{i}}\left\{\sum_{i=1}^{s}\left(\operatorname{trace}\left(\Gamma_{i}\right)+\operatorname{trace}\left(\Phi_{i}\right)\right)\right\} \\
\text { s.t. }(37)
\end{array}\right.
$$

Denote $\Gamma_{i}^{*}, \Phi_{i}^{*}$ as an optimal solution of (41), and

$$
\Xi_{i}^{*}=\left[\begin{array}{cc}
\Gamma_{i}^{*} & \mathbf{0}_{n \times n} \\
\mathbf{0}_{n \times n} & \Phi_{i}^{*}
\end{array}\right], i=\overline{1, s}
$$

At time $k$, consider the following optimization problem

$$
\begin{aligned}
& \min _{v(k)} \max _{i=1, s}\left\{\frac{1}{2} x(k)^{T} \Gamma_{i}^{*} x(k)+\frac{1}{2} v(k)^{T} \Phi_{i}^{*} v(k)\right\} \\
& \text { s.t. }\left[x(k)^{T} v(k)^{T}\right]^{T} \in \bigcap_{i=1}^{s} \mathcal{E}\left(P_{i}\right)
\end{aligned}
$$

Let $v^{*}(k)$ be the solution of (42). The control signal at time $k$ is

$$
u(k)=K x(k)+(L-K) v^{*}(k)
$$


Theorem 4: Assuming feasibility at the initial state, the optimization based controller (43) guarantees recursive feasibility and robust asymptotic stability.

Proof: For a given feasible state $x(k)$, the set $\bigcap_{i=1}^{s} \mathcal{E}\left(P_{i}\right)$ is not empty for $v(k)$. Hence the optimization problem (42) is feasible at time $k \cdot \bigcap_{i=1}^{s} \mathcal{E}\left(P_{i}\right)$ is robustly invariant and constraintadmissible (14), (4) under the control law (43). Hence $z(k+1)=\left[\begin{array}{c}x(k+1) \\ v(k+1)\end{array}\right] \in \bigcap_{i=1}^{s} \mathcal{E}\left(P_{i}\right)$. As consequence, recursive feasibility is guaranteed.

For the robust asymptotic stability proof, consider the following Lyapunov function candidate

$$
V\left(k, z^{*}(k)\right)=z^{*}(k)^{T}\left(\sum_{i=1}^{s} \lambda_{i}(k) \Xi_{i}^{*}\right) z^{*}(k)
$$

where $z^{*}(k)=\left[\begin{array}{ll}x(k)^{T} & v^{*}(k)^{T}\end{array}\right]$. One obtains $z(k+1)$ by applying (43) to (1). Using (36), one gets, $\forall \lambda_{i_{1}}(k+1) \in \Lambda$

$$
\begin{aligned}
& z(k+1)^{T}\left(\sum_{i_{1}=1}^{s} \lambda_{i_{1}}(k+1) \Xi_{i_{1}}^{*}\right) z(k+1)-z^{*}(k)^{T}\left(\sum_{i=1}^{s} \lambda_{i}(k) \Xi_{i}^{*}\right) z^{*}(k) \\
& \leq-x(k)^{T} Q x(k)-u(k)^{T} R u(k)
\end{aligned}
$$

Thus

$$
\begin{gathered}
\max _{i_{1}=1, s}\left\{z(k+1)^{T} \Xi_{i_{1}}^{*} z(k+1)\right\}-z^{*}(k)^{T}\left(\sum_{i=1}^{s} \lambda_{i}(k) \Xi_{i}^{*}\right) z^{*}(k) \\
\leq-x(k)^{T} Q x(k)-u(k)^{T} R u(k)
\end{gathered}
$$

Solving the problem (42) at time $k+1$ yields $z^{*}(k+1)$ such that

$$
\max _{i_{1}=\overline{1, s}}\left\{z^{*}(k+1)^{T} \Xi_{i_{1}}^{*} z^{*}(k+1)\right\} \leq \max _{i_{1}=\overline{1, s}}\left\{z(k+1)^{T} \Xi_{i_{1}}^{*} z(k+1)\right\}
$$

Combining (45), (46), and note that

$$
z^{*}(k+1)^{T}\left(\sum_{i_{1}=1}^{s} \lambda_{i_{1}}(k+1) \Xi_{i_{1}}^{*}\right) z^{*}(k+1) \leq \max _{i_{1}=\overline{1, s}}\left\{z^{*}(k+1)^{T} \Xi_{i_{1}}^{*} z^{*}(k+1)\right\}
$$

one obtains

$$
\begin{aligned}
& z^{*}(k+1)^{T}\left(\sum_{i_{1}=1}^{s} \lambda_{i_{1}}(k+1) \Xi_{i_{1}}^{*}\right) z^{*}(k+1)-z^{*}(k)^{T}\left(\sum_{i=1}^{s} \lambda_{i}(k) \Xi_{i}^{*}\right) z^{*}(k) \\
& \quad \leq-x(k)^{T} Q x(k)-u(k)^{T} R u(k)
\end{aligned}
$$

Hence $V(k, z(k))$ is a Lyapunov function of the closed-system under the control law (43). In other words, robust asymptotic stability is assured [16].

Remark 3: Using the simulation results it is observed that the online computational complexity is reduced if the same $\Phi_{i}^{*}$ can be used for a set of vertices. Note that no constraints are imposed 
on $\Gamma_{i}^{*} . \Gamma_{i}^{*}$ can be different for different vertices. In the extreme case, i.e., $\Phi_{i}^{*}=\Phi^{*}, \forall i=\overline{1, s}$, one has, for the cost function (42)

$$
\begin{gathered}
\min _{v(k)} \max _{i=\overline{1, s}}\left\{\frac{1}{2} x(k)^{T} \Gamma_{i}^{*} x(k)+\frac{1}{2} v(k)^{T} \Phi^{*} v(k)\right\} \\
=\min _{v(k)}\left\{\frac{1}{2} v(k)^{T} \Phi^{*} v(k)\right\}+\max _{i=\overline{1, s}}\left\{\frac{1}{2} x(k)^{T} \Gamma_{i}^{*} x(k)\right\}
\end{gathered}
$$

The term $\max _{i=1, s}\left\{\frac{1}{2} x(k)^{T} \Gamma_{i}^{*} x(k)\right\}$ can be removed, since it does not influence the optimal argument. (42) becomes

$$
\begin{aligned}
& \min _{v(k)}\left\{\frac{1}{2} v(k)^{T} \Phi^{*} v(k)\right\} \\
& \text { s.t. }\left[x(k)^{T} v(k)^{T}\right]^{T} \in \bigcap_{i=1}^{s} \mathcal{E}\left(P_{i}\right)
\end{aligned}
$$

\section{Online Optimization Problem}

By using the equivalent epigraph representation [17] of (42), it can be shown that (42) can be converted into a convex quadratically constrained quadratic program (QCQP). Hence the solution can be obtained by using, e.g., the interior point method [18]. In general, QCQP can be solved much more efficient than SDP [17].

In the following another way to solve (42) is provided. Note that problem (42) has a very nice geometrical interpretation. The solution to (42) defines the shortest distance of the intersection of $s$ ellipsoids from the origin with respect to a min-max norm. To see this, for a given $x(k)$ define $\Omega_{v}$ as the feasible set, to which $v$ must belong. $\Omega_{v}$ is the following set

$$
\Omega_{v}=\left\{v(k) \in \mathbb{R}^{n}:\left[x(k)^{T} v(k)^{T}\right]^{T} \in \bigcap_{i=1}^{s} \mathcal{E}\left(P_{i}\right)\right\}
$$

i.e., $\Omega_{v}$ is the intersection of $s$ ellipsoids. If the origin is contained in $\Omega_{v}$, then $v^{*}(k)=0$ is the solution of (42). In this case, (43) becomes $u(k)=K x(k)$, i.e., the robust optimal LQ control law. Otherwise, if the origin is not contained in $\Omega_{v}$, then $v^{*}(k)$ must lie on the boundary of $\Omega_{v}$ that is closest to the origin.

Problem (42) can be decomposed into $s$ parallel optimization sub-problems, $i=\overline{1, s}$

$$
\begin{aligned}
& \min _{v(k)}\left\{\frac{1}{2} v(k)^{T} \Phi_{i}^{*} v(k)\right\}, \\
& \text { s.t. }\left[x(k)^{T} v(k)^{T}\right]^{T} \in \bigcap_{i=1}^{s} \mathcal{E}\left(P_{i}\right)
\end{aligned}
$$

Let $v_{i}^{*}(k)$ be the solution of (48), $i=\overline{1, s}$. Define, $i_{1}=\overline{1, s}$

$$
g_{i_{1} i}=\frac{1}{2} x(k)^{T} \Gamma_{i_{1}}^{*} x(k)+\frac{1}{2} v_{i}^{*}(k)^{T} \Phi_{i}^{*} v_{i}^{*}(k)
$$


Define also an index $i^{*}$ such that

$$
\max _{i_{1}=\overline{1, s}}\left\{g_{i_{1} i^{*}}\right\} \leq g_{i^{*} i^{*}}
$$

Clearly, $v_{i^{*}}^{*}(k)$ is the solution of (42). Now a way to solve (48) is presented. Rewrite (48) as

$$
\begin{array}{ll}
\min _{f(k)}\left\{\begin{array}{l}
\left\{\frac{1}{2} f(k)^{T} f(k)\right\}, \\
\text { s.t. }\left\{\begin{array}{ll}
x(k)^{T} & f(k)^{T}
\end{array}\right] Q_{1}\left[\begin{array}{c}
x(k) \\
f(k)
\end{array}\right] \leq 1, \\
\vdots \\
{\left[\begin{array}{ll}
x(k)^{T} & f(k)^{T}
\end{array}\right] Q_{s}\left[\begin{array}{c}
x(k) \\
f(k)
\end{array}\right] \leq 1}
\end{array}\right.
\end{array}
$$

where $f(k)=\Phi_{c i} v(k), \Phi_{c i}^{T} \Phi_{c i}=\Phi_{i}^{*}, i=\overline{1, s}$, and,

$$
Q_{i}=\left[\begin{array}{c}
\mathbf{I}_{n \times n} \\
\left(\Phi_{c i}^{-1}\right)^{T}
\end{array}\right] P_{i}^{-1}\left[\begin{array}{ll}
\mathbf{I}_{n \times n} & \Phi_{c i}^{-1}
\end{array}\right]
$$

The cost function of (50) is strongly convex, and the feasible set is the intersection of $s$ closed convex sets. This is a classical optimization problem that is tackled by many authors, e.g., [19], [20]. In the present paper, the algorithm in Han and Lou [19] is applied. This method splits up the computation into $s$ parallel sub-problems. The required matrix operations are extremely simple. The main idea of this method is to solve the dual problem of (50) using parallel computations in an iterative scheme. The application of the method [19] to our problem is summarized in the following. We use $q$ as iteration counter of the method. A superscript $(q)$ is used to denote the values of variables calculated at iteration $q$. Define $f^{(0)}=y_{1}^{(0)}=\ldots=y_{s}^{(0)}=0$. Let $\rho$ be a sufficiently large number. For $q=1,2, \ldots$, we perform the following calculations

1) For $i=\overline{1, s}$, find $f_{i}^{(q)}$ that solves

$$
\begin{aligned}
& \min _{f_{i}} \frac{1}{2}\left(f_{i}+\rho y_{i}^{(q-1)}-f^{(q-1)}\right)^{T}\left(f_{i}+\rho y_{i}^{(q-1)}-f^{(q-1)}\right), \\
& \text { s.t. }\left[\begin{array}{ll}
x(k)^{T} & f_{i}^{T}
\end{array}\right] Q_{i}\left[\begin{array}{c}
x(k) \\
f_{i}
\end{array}\right] \leq 1
\end{aligned}
$$

To solve (51), the algorithm in [21] is employed. This method requires only to find the unique positive root of a well behave polynomial equation [2]. The solution can be found by a univariate Newton-Raphson procedure with quadratic convergence rate.

2) Assign

$$
y_{i}^{(q)}=y_{i}^{(q-1)}+\frac{1}{\rho}\left(f_{i}^{(q)}-f^{(q-1)}\right)
$$


3) Set $f^{(q)}=y_{1}^{(q)}+\ldots+y_{s}^{(q)}$

The design parameter $\rho$ must be sufficiently large. With $\rho \geq \frac{s}{\rho^{\prime}}$, the method [19] will converge with the following contraction rate

$$
\left(f^{(q)}-f^{(q-1)}\right)^{T}\left(f^{(q)}-f^{(q-1)}\right) \leq \frac{s}{2 \rho^{\prime}}\left(y_{i}^{(q)}-y_{i}^{(q-1)}\right)^{T}\left(y_{i}^{(q)}-y_{i}^{(q-1)}\right)
$$

For a quadratic cost function, we can choose $\rho^{\prime}$ as the smallest eigenvalue of the Hessian matrix, i.e., $\rho^{\prime}=1$ in our case. Therefore $\rho=s$.

Remark 4: With $q=1$, the optimization problem (50) with only one active ellipsoidal constraint is the same as (51). If there exists index $i$ such that, for $q=1$,

$$
\left[x(k)^{T}\left(f_{i}^{(q)}\right)^{T}\right] Q_{p}\left[\begin{array}{c}
x(k) \\
f_{i}^{(q)}
\end{array}\right] \leq 1, \forall i=\overline{1, s}
$$

then $f^{*}=f_{i}^{(q)}$ is the solution of (50).

To sum up, the proposed control policy with linear feedback consists of two stages: offline stage and online stage.

\section{Offline Stage}

1: Select the matrix gain $L$.

2: Obtain $P_{i}, \forall i=\overline{1, s}$ by solving (34).

3: Obtain $\Gamma_{i}^{*}, \Phi_{i}^{*}, \forall i=\overline{1, s}$ by solving (41).

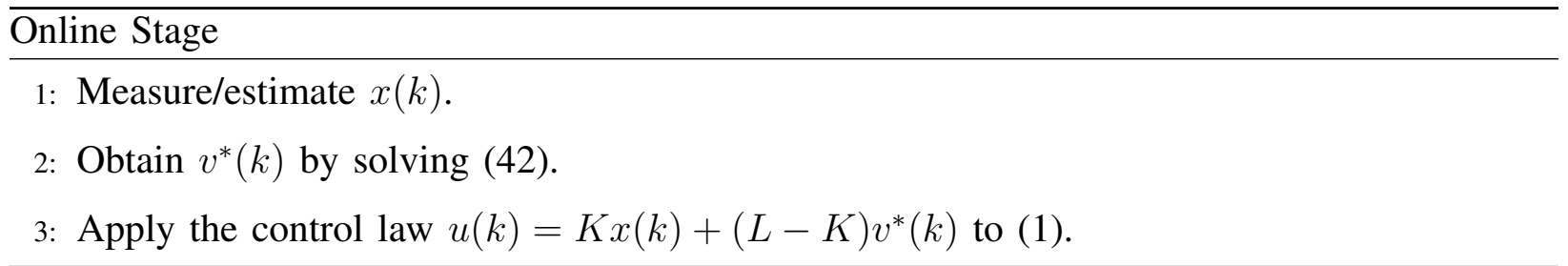

\section{PRediction Dynamics with SATURATEd FEedback}

\section{A. Prediction Dynamics}

As written in Introduction, despite an efficient online computation, the control signal of the prediction dynamics based MPC method with linear feedback hits rarely the constraints. Hence the time to regulate the plant to the origin is much longer than, e.g., by time-optimal control. To overcome this problem, a new prediction dynamics based MPC method with saturated feedback 
was considered in [2]. With the new algorithm, the control range is fully exploited, and the performance can be significantly improved. In addition, the domain of attraction of the new control law can be as large as that of any static saturated state feedback law, $L \in \mathbb{R}^{m \times n}$

$$
u(k)=\operatorname{sat}(L x(k))
$$

However the method in [2] is still based on common quadratic functions to characterize the domain of attraction as well as to calculate the upper bound of the cost function. The main aim of this section is to present a way to reduce the convervativeness of [2]. In addition, a particular choice of the dynamic controller parameters is also proposed. To this aim, consider the following saturated dynamic control law

$$
u(k)=\operatorname{sat}(K x(k)+(L-K) v(k))
$$

where $v(k) \in \mathbb{R}^{n}$ is the controller state. The saturation function $\operatorname{sat}(u): \mathbb{R}^{m} \rightarrow \mathbb{R}^{m}$ is defined as

$$
\begin{aligned}
& \operatorname{sat}(u)=\left[\operatorname{sat}\left(u_{1}\right) \operatorname{sat}\left(u_{2}\right) \ldots \operatorname{sat}\left(u_{m}\right)\right]^{T} \\
& \operatorname{sat}\left(u_{j}\right)= \begin{cases}-1, & \text { if } u_{j} \leq-1, \\
u_{j}, & \text { if }-1 \leq u_{j} \leq 1, \\
1, & \text { if } 1 \leq u_{j}\end{cases}
\end{aligned}
$$

Note that using (54), (55), the input constraints (4) are automatically satisfied. Note also that if $v(k)=0$, then (54) is the robust optimal saturated LQ controller

$$
u(k)=\operatorname{sat}(K x(k))
$$

In the following we recall the linear differential inclusion (LDI) modeling framework, which was proposed in [8]. It will be used to model the saturation nonlinearity.

Define $\mathcal{D}$ as the set of $m \times m$ diagonal matrix whose diagonal elements are either 0 or 1 . For example, if $m=2$, then

$$
\mathcal{D}=\left\{\left[\begin{array}{ll}
1 & 0 \\
0 & 1
\end{array}\right],\left[\begin{array}{ll}
1 & 0 \\
0 & 0
\end{array}\right],\left[\begin{array}{ll}
0 & 0 \\
0 & 1
\end{array}\right],\left[\begin{array}{ll}
0 & 0 \\
0 & 0
\end{array}\right]\right\}
$$

There are $2^{m}$ elements in $\mathcal{D}$. Define $E_{r}, r=\overline{1,2^{m}}$ as an element in $\mathcal{D}$. Define also $E_{r}^{-}=$ $\mathbf{I}_{m \times m}-E_{r}$. Clearly, $E_{r}^{-}$is also an element of $\mathcal{D}$.

Lemma 3: [8] Let $S_{v}, S_{o} \in \mathbb{R}^{m \times n}$. One has, $\forall x(k) \in \mathcal{L}\left(S_{o}\right)$

$$
\operatorname{sat}\left(S_{v} x(k)\right)=\sum_{r=1}^{2^{m}} \alpha_{r}(k)\left(E_{r} S_{v}+E_{r}^{-} S_{o}\right) x(k)
$$


where $\sum_{r=1}^{2^{m}} \alpha_{r}(k)=1, \alpha_{r}(k) \geq 0$.

For example, if $m=2$, we have

$$
\left[\begin{array}{c}
\operatorname{sat}\left(S_{v, 1} x\right) \\
\operatorname{sat}\left(S_{v, 2} x\right)
\end{array}\right]=\alpha_{1}\left[\begin{array}{c}
S_{v, 1} x \\
S_{v, 2} x
\end{array}\right]+\alpha_{2}\left[\begin{array}{c}
S_{v, 1} x \\
S_{o, 2} x
\end{array}\right]+\alpha_{3}\left[\begin{array}{c}
S_{o, 1} x \\
S_{v, 2} x
\end{array}\right]+\alpha_{4}\left[\begin{array}{c}
S_{o, 1} x \\
S_{o, 2} x
\end{array}\right]
$$

Remark 5: For simplicity the LDI modeling framework in [8] is used. However, the approach in the paper can be straightforwardly extended with the LDI framework in [22]. Compared with [8], the main advantage of [22] is that the conservativeness is reduced. However, it comes with a cost of higher computational complexity.

Using Lemma 3, a way to model the system (1) with the saturated control law (53) and (56) is proposed. The obtained models are then used to design the prediction dynamics with the control law (54). Since the way to model (1) with (53), or (1) with (56) are the same, only the one with (53) is shown here. Using Lemma $3, \forall x(k) \in \mathcal{L}(H)$

$$
u(k)=\operatorname{sat}(L x(k))=\sum_{r=1}^{2^{m}} \alpha_{r}(k)\left(E_{r} L+E_{r}^{-} H\right) x(k)
$$

where $H \in \mathbb{R}^{m \times n}$ is an unknown matrix that will be treated as a decision variable, $\sum_{r=1}^{2^{m}} \alpha_{r}(k)=$ $1, \alpha_{r}(k) \geq 0$.

Consider the polytope $\mathcal{S}$, the vertices of which are given by taking all possible combinations of $\left\{A_{i}+B_{i} E_{r} L+B_{i} E_{r}^{-} H\right\}$, where $i=\overline{1, s}, r=\overline{1,2^{m}}$. There are $s 2^{m}$ vertices of $\mathcal{S}$.

Theorem 5: The closed-loop system matrix of (1), (53) can be expressed as a convex combination of the vertices of $\mathcal{S}, \forall x(k) \in \mathcal{L}(H)$.

Proof: Substituting (58) into (1), one gets

$$
\begin{aligned}
x(k+1) & =\left(\sum_{i=1}^{s} \lambda_{i}(k) A_{i}+\sum_{i=1}^{s} \lambda_{i}(k) B_{i} \sum_{r=1}^{2^{m}} \alpha_{r}(k)\left(E_{r} L+E_{r}^{-} H\right)\right) x(k) \\
& =\sum_{i=1}^{s} \lambda_{i}(k)\left(A_{i}+\sum_{r=1}^{2^{m}} \alpha_{r}(k) B_{i}\left(E_{r} L+E_{r}^{-} H\right)\right) x(k)
\end{aligned}
$$

or equivalently

$$
x(k+1)=\sum_{i=1}^{s} \sum_{r=1}^{2^{m}} \lambda_{i}(k) \alpha_{r}(k)\left(A_{i}+B_{i} E_{r} L+B_{i} E_{r}^{-} H\right) x(k)
$$

Define, $\forall i=\overline{1, s}, \forall r=\overline{1,2^{m}}$

$$
\mathbf{A}_{p}=A_{i}+B_{i} E_{r} L, \mathbf{B}_{p}=B_{i} E_{r}^{-}, \forall p=\overline{1, s 2^{m}}
$$


and $\zeta_{p}(k)=\lambda_{i}(k) \alpha_{r}(k)$. For example, if $s=2, m=1$, then

$$
\begin{aligned}
& \mathbf{A}_{1}=A_{1}+B_{1} L, \mathbf{B}_{1}=\mathbf{0}, \mathbf{A}_{2}=A_{1}, \mathbf{B}_{2}=B 1, \\
& \mathbf{A}_{3}=A_{2}+B_{2} L, \mathbf{B}_{3}=\mathbf{0}, \mathbf{A}_{4}=A_{2}, \mathbf{B}_{4}=B 2, \\
& \zeta_{1}=\lambda_{1} \alpha_{1}, \zeta_{2}=\lambda_{1} \alpha_{2}, \zeta_{3}=\lambda_{2} \alpha_{1}, \zeta_{4}=\lambda_{2} \alpha_{2}
\end{aligned}
$$

Rewrite (59) as

$$
x(k+1)=\sum_{p=1}^{s 2^{m}} \zeta_{p}(k)\left(\mathbf{A}_{p}+\mathbf{B}_{p} H\right) x(k)
$$

Using the fact that

$$
\sum_{p=1}^{s 2^{m}} \zeta_{p}(k)=\sum_{i=1}^{s} \sum_{r=1}^{2^{m}} \lambda_{i}(k) \alpha_{r}(k)=\sum_{i=1}^{s} \lambda_{i}(k) \sum_{r=1}^{2^{m}} \alpha_{r}(k)=1
$$

it follows that the system matrix (61) can be expressed as a convex combination of vertices of $\mathcal{S}$.

The following theorem concerns conditions for a set to be robustly invariant and constraintadmissible for (1), (4) under the control law (53).

Theorem 6: Suppose that matrices $W_{p} \in \mathbb{R}^{n \times n}, W_{p} \succ 0, \forall p=\overline{1, s 2^{m}}, G \in \mathbb{R}^{n \times n}, Y \in \mathbb{R}^{m \times n}$ satisfy the following LMIs

$$
\begin{aligned}
& {\left[\begin{array}{cc}
W_{p_{1}} & \left(\mathbf{A}_{p} G+\mathbf{B}_{p} Y\right) \\
(*) & G+G^{T}-W_{p}
\end{array}\right] \succeq 0, \forall p, \forall p_{1}=\overline{1, s 2^{m}}} \\
& {\left[\begin{array}{cc}
1 & Y_{j} \\
Y_{j}^{T} & G+G^{T}-W_{p_{2}}
\end{array}\right] \succeq 0, \exists p_{2} \in \overline{1, s 2^{m}}, \forall j=\overline{1, m}} \\
& 1-f_{l} W_{p_{3}} f_{l}^{T} \geq 0, \exists p_{3} \in \overline{1, s 2^{m}}, \forall l=\overline{1, n_{c}}
\end{aligned}
$$

where $Y_{j}$ is the $j$ th row of $Y$. Then $\bigcap_{p=1}^{s 2^{m}} \mathcal{E}\left(W_{p}\right)$ is robustly invariant for

$$
x(k+1)=A(k) x(k)+B(k) \operatorname{sat}(L x(k))
$$

and constraint-admissible with respect to (4).

Proof: Using Lemma 1, condition (64) guarantees that $\mathcal{E}\left(W_{p_{3}}\right) \in \mathcal{L}\left(f_{l}\right)$. Because $\bigcap_{p=1}^{s 2^{m}} \mathcal{E}\left(W_{p}\right) \subseteq$ $\mathcal{E}\left(W_{p_{3}}\right)$, it follows $\bigcap_{p=1}^{s 2^{m}} \mathcal{E}\left(W_{p}\right) \subseteq \mathcal{L}\left(f_{l}\right), \forall l=\overline{1, n_{c}}$. Hence the state constraints are satisfied.

$\forall x \in \mathcal{L}(H)$, the closed-loop system (1), (53) can be modeled by (61). Using Lemma 1 , $\forall j=\overline{1, m}$, if $\exists p_{2} \in \overline{1, s 2^{m}}$ such that

$$
1-H_{j} W_{p_{2}} H_{j}^{T} \geq 0
$$


then $\mathcal{E}\left(W_{p_{2}}\right) \in \mathcal{L}\left(H_{j}\right)$. With $H_{j}=Y_{j} G^{-1}$, condition (65) becomes

$$
1-Y_{j} G^{-1} W_{p_{2}}\left(G^{-1}\right)^{T} Y_{j}^{T} \geq 0
$$

Thus, with Schur complement

$$
\left[\begin{array}{cc}
1 & Y_{j} \\
Y_{j}^{T} & G^{T} W_{p_{2}}^{-1} G
\end{array}\right] \succeq 0
$$

Using Lemma 2, if (63) holds then (66) is satisfied. Since $\bigcap_{p=1}^{s 2^{m}} \mathcal{E}\left(W_{p}\right) \subseteq \mathcal{E}\left(W_{p_{2}}\right)$, condition (63) assures that $\forall x \in \bigcap_{p=1}^{s 2^{m}} \mathcal{E}\left(W_{p}\right)$, system (1), (53) can be modeled using (61). It remains to show that $\bigcap_{p=1}^{s 2^{m}} \mathcal{E}\left(W_{p}\right)$ is robustly invariant. Using Lemma 2, if (62) holds then

$$
\left[\begin{array}{cc}
W_{p_{1}} & \left(\mathbf{A}_{p} G+\mathbf{B}_{p} Y\right) \\
(*) & G^{T} W_{p}^{-1} G
\end{array}\right] \succeq 0, \forall p, \forall p_{1}=\overline{1, s 2^{m}}
$$

Pre- and post-multiplication of (67) by

$$
\left[\begin{array}{cc}
W_{p_{1}}^{-1} & \mathbf{0}_{n \times n} \\
\mathbf{0}_{n \times n} & \left(G^{-1}\right)^{T}
\end{array}\right],\left[\begin{array}{cc}
W_{p_{1}}^{-1} & \mathbf{0}_{n \times n} \\
\mathbf{0}_{n \times n} & G^{-1}
\end{array}\right]
$$

one obtains, $H=Y G^{-1}$

$$
\left[\begin{array}{cc}
W_{p_{1}}^{-1} & W_{p_{1}}^{-1}\left(\mathbf{A}_{p}+\mathbf{B}_{p} H\right) \\
(*) & W_{p}^{-1}
\end{array}\right] \succeq 0, \forall p, \forall p_{1}=\overline{1, s 2^{m}}
$$

For each $p_{1}$, multiply the corresponding $p=\overline{1, s 2^{m}}$ by $\zeta_{p}(k)$, and sum to obtain

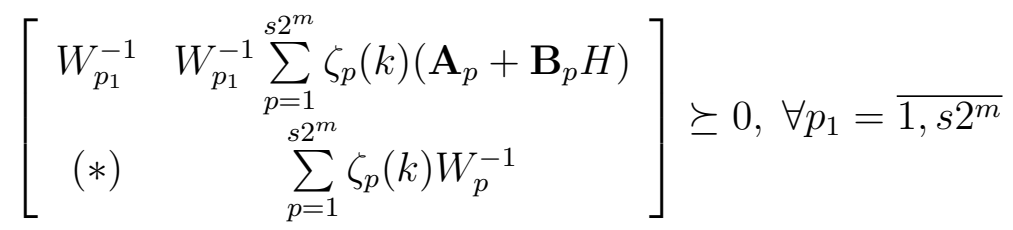

Thus, with Schur complement, $p_{1}=\overline{1, s 2^{m}}$

$$
\left(\sum_{p=1}^{s 2^{m}} \zeta_{p}(k) W_{p}^{-1}\right)-\left(\sum_{p=1}^{s 2^{m}} \zeta_{p}(k)\left(\mathbf{A}_{p}+\mathbf{B}_{p} H\right)\right)^{T} W_{p_{1}}^{-1}\left(\sum_{p=1}^{s 2^{m}} \zeta_{p}(k)\left(\mathbf{A}_{p}+\mathbf{B}_{p} H\right)\right) \succeq 0
$$

Pre- and post-multiplication of (68) with $x(k)^{T}$ and $x(k)$, and note that

$$
x(k+1)=\left(\sum_{p=1}^{s 2^{m}} \zeta_{p}(k)\left(\mathbf{A}_{p}+\mathbf{B}_{p} H\right)\right) x(k)
$$


one gets

$$
\left(\sum_{p=1}^{s 2^{m}} \zeta_{p}(k) x(k)^{T} W_{p}^{-1} x(k)\right)-x(k+1)^{T} W_{p_{1}}^{-1} x(k+1) \geq 0
$$

If $x(k) \in \bigcap_{p=1}^{s 2^{m}} \mathcal{E}\left(W_{p}\right)$, then $x(k)^{T} W_{p}^{-1} x(k) \leq 1$. If follows that $\sum_{p=1}^{s 2^{m}} \zeta_{p}(k) x(k)^{T} W_{p}^{-1} x(k) \leq 1$. As consequence, $x(k+1)^{T} W_{p_{1}}^{-1} x(k+1) \leq 1$, or $x(k+1) \in \mathcal{E}\left(W_{p_{1}}\right), \forall p_{1}=\overline{1, s 2^{m}}$. In other words, $x(k+1) \in \bigcap_{p=1}^{s 2^{m}} \mathcal{E}\left(W_{p}\right)$. Hence $\bigcap_{p=1}^{s 2^{m}} \mathcal{E}\left(W_{p}\right)$ is robustly invariant.

In general, one would like to maximize the size of $\bigcap_{p=1}^{s 2^{m}} \mathcal{E}\left(W_{p}\right)$. This can be done as in Section II-B, i.e., maximize $\bigcap_{p=1}^{s 2^{m}} \mathcal{E}\left(W_{p}\right)$ with respect to some shape reference sets. Alternatively, the following SDP problem can be used to optimize the volume of $\bigcap_{p=1}^{s 2^{m}} \mathcal{E}\left(W_{p}\right)$.

$$
\max _{W_{p}, G, Y}\left\{\sum_{p=1}^{s 2^{m}} \log \operatorname{det}\left(W_{p}\right)\right\}
$$

$$
\text { s.t. }(62),(63),(64)
$$

Denote $W_{p}^{*}, p=\overline{1, s 2^{m}}, G^{*}, Y^{*}$ and $H=Y^{*}\left(G^{*}\right)^{-1}$ as an optimal solution of (69). $W_{p}^{*}$ will be used to show a theoretical property of the new control law (54), while $H$ is for designing the prediction dynamics.

Analogously, by solving a corresponding SDP problem, one can obtain an auxiliary matrix gain $V \in \mathbb{R}^{m \times n}$ for the saturated controller (56) such that the domain of attraction of the closedloop system (1), (56) is maximized. Consider now the saturated dynamic control law (54). Using Lemma 3, one gets

$$
\operatorname{sat}(K x+(L-K) v)=\sum_{r=1}^{2^{m}} \alpha_{r}\left\{E_{r}(K x+(L-K) v)+E_{r}^{-}(V x+(H-V) v)\right\}
$$

$\forall x$, and $\forall v$ such that

$$
\left[\begin{array}{ll}
x^{T} & v^{T}
\end{array}\right]^{T} \in \mathcal{L}(\mathbf{H})
$$

where $\mathbf{H}=[V H-V]$. By using the same procedure as the one to prove theorem 5 , it can be shown that the closed-loop system (1), (54) can be rewritten as, $\forall x, \forall v$ satisfying (71)

$$
x(k+1)=\sum_{p=1}^{s 2^{m}} \zeta_{p}(k)\left(\mathbf{A}_{x, p} x(k)+\mathbf{B}_{x, p} v(k)\right)
$$

where $\zeta_{p}(k)=\lambda_{i}(k) \alpha_{r}(k), \forall i=\overline{1, s}, r=\overline{1,2^{m}}, p=\overline{1, s 2^{m}}$, and

$$
\left\{\begin{array}{l}
\mathbf{A}_{x, p}=A_{i}+B_{i} E_{r} K+B_{i} E_{r}^{-} V \\
\mathbf{B}_{x, p}=B_{i} E_{r}(L-K)+B_{i} E_{r}^{-}(H-V)
\end{array}\right.
$$


Assuming $v(k)$ is the output of the following auxiliary system

$$
v(k+1)=\sum_{p=1}^{s 2^{m}} \zeta_{p}(k)\left(\mathbf{A}_{p}+\mathbf{B}_{p} H\right) v(k)
$$

where $\mathbf{A}_{p}, \mathbf{B}_{p}$ are given in (60). Note that the dynamics (73) depend not only on the polytopic uncertainty (2), but also on the input saturations. Combining (72), (73), one obtains

$$
z(k+1)=\sum_{p=1}^{s 2^{m}} \zeta_{p}(k) \mathcal{A}_{c, p} z(k)
$$

where

$$
z(k)=\left[\begin{array}{l}
x(k) \\
v(k)
\end{array}\right], \mathcal{A}_{c, p}=\left[\begin{array}{cc}
\mathbf{A}_{x, p} & \mathbf{B}_{x, p} \\
\mathbf{0}_{n} & \mathbf{A}_{p}+\mathbf{B}_{p} H
\end{array}\right], \forall p=\overline{1, s 2^{m}}
$$

Define $\Omega_{c, z}$ as a robustly invariant and constraint-admissible set for (74), for the state constraints (4), and for (71). Define also $\Omega_{c, x}$ as the projection of $\Omega_{c, z}$ onto the $x$-space. The following corollary holds

Corollary 1: $\Omega_{c, z}$ can be optimized in such a way that $\bigcap_{p=1}^{s 2^{m}} \mathcal{E}\left(W_{p}^{*}\right) \subseteq \Omega_{c, x}$.

Proof: It is omitted here, since it follows the same lines as the proof of Theorem 1.

Corollary 1 states that the domain of attraction of the control law (54) can be as large as that of any static saturated state feedback law. Note that for simplicity the intersection of ellipsoids $\bigcap_{t=1}^{s 2^{m}} \mathcal{E}\left(W_{p}^{*}\right)$ is used as the domain of attraction for the closed-loop system (1), (53). However, it is clear that this set can be any kind of set.

\section{B. Domain of Attraction, Cost Function and Control Law}

Once the prediction dynamics are designed, the next steps are: i) calculate the domain of attraction, ii) estimate the upper bound of the cost function $J(k)$, iii) minimize the obtained upper bound online.

Similarly to Theorem 2, the following corollary establishes the theoretical support of the algorithm proposed to obtain an estimation of the domain of attraction for (74).

Corollary 2: Suppose that matrices $P_{c, p} \in \mathbb{R}^{2 n \times 2 n}, P_{c, p} \succ 0, \forall p=\overline{1, s 2^{m}}$ satisfy the following LMIs

$$
\begin{aligned}
& {\left[\begin{array}{cc}
P_{c, p_{1}} & \mathcal{A}_{c, p} P_{c, p} \\
P_{c, p} \mathcal{A}_{c, p}^{T} & P_{c, p}
\end{array}\right] \succeq 0, \forall p_{1}, \forall p=\overline{1, s 2^{m}}} \\
& 1-\mathbf{H}_{j} P_{c, p_{2}} \mathbf{H}_{j} \geq 0, \exists p_{2} \in \overline{1, s 2^{m}}, \forall j=\overline{1, m} \\
& 1-\mathbf{F}_{l} P_{c, p_{3}} \mathbf{F}_{l}^{T} \geq 0, \exists p_{3} \in \overline{1, s 2^{m}}, \forall l=\overline{1, n_{c}}
\end{aligned}
$$


where $\mathbf{H}_{j}$ is the $j$ th row of $\mathbf{H}$. Then the set $\bigcap_{p=1}^{s 2^{m}} \mathcal{E}\left(P_{c, p}\right)$ is robustly invariant for (74), and constraints admissible with respect to the state constraints (4), and to (71).

Proof: It is omitted here.

Once robust invariance and constraint admissible conditions are expressed as LMI constraints, the size of $\bigcap_{p=1}^{s 2^{m}} \mathcal{E}\left(P_{c, p}\right)$ can be maximized as in Section II-B. Details are not considered further.

Our next step is to calculate the upper bound of the cost function $J(k)$. Consider the following time-varying quadratic function

$$
V_{c}(k, z(k))=z(k)^{T}\left(\sum_{p=1}^{s 2^{m}} \zeta_{p}(k) \Xi_{c, p}\right) z(k)
$$

where $\Xi_{c, p} \in \mathbb{R}^{2 n \times 2 n}, \Xi_{c, p} \succeq 0, \forall p=\overline{1, s 2^{m}}$ are chosen to satisfy

$$
\begin{aligned}
& x(k+t)^{T} Q x(k+t)+u(k+t)^{T} R u(k+t) \leq \\
& \leq V_{c}(k, z(k))-V_{c}(k+1, z(k+1))
\end{aligned}
$$

By summing (79) from $t=0$ to $t=\infty$, one can show that $J(k) \leq V_{c}(k, z(k))$. Or equivalently $V_{c}(k, z(k))$ is an upper bound of $J(k)$.

Define, $\forall i=\overline{1, s}, \forall r=\overline{1,2^{m}}, \forall p=\overline{1, s 2^{m}}$

$$
\mathbf{R}_{p}=\left(E_{r} \mathbf{K}+E_{r}^{-} \mathbf{H}\right)^{T} R_{i}\left(E_{r} \mathbf{K}+E_{r}^{-} \mathbf{H}\right)
$$

where $R_{i}=R, \forall i=\overline{1, s}$. The following corollary concerns the existence of $\Xi_{c, p}$.

Corollary 3: There exist $\Xi_{c, p}, \forall p=\overline{1, s 2^{m}}$ satisfying (79), if and only if the following LMIs hold, $\forall p, \forall p_{1}=\overline{1, s 2^{m}}$

$$
\left[\begin{array}{cc}
\left(\Xi_{c, p}-\mathbf{Q}-\mathbf{R}_{p}\right) & \mathcal{A}_{c, p}^{T} \Xi_{c, p_{1}} \\
\Xi_{c, p_{1}} \mathcal{A}_{c, p} & \Xi_{c, p_{1}}
\end{array}\right] \succeq 0
$$

where $\mathrm{Q}$ is defined in (38).

Proof: The proof is omitted since it is almost the same as the one of Theorem 3.

We are generally looking for $\Xi_{c, p}$ in the diagonal form, $p=\overline{1, s 2^{m}}$

$$
\Xi_{c, p}=\left[\begin{array}{cc}
\Gamma_{c, p} & \mathbf{0}_{n \times n} \\
\mathbf{0}_{n \times n} & \Phi_{c, p}
\end{array}\right]
$$

The optimal $\Xi_{c, p}$ can be found by solving the following SDP problem

$$
\min _{\Gamma_{c, p}, \Phi_{c, p}}\left\{\sum_{p=1}^{s 2^{m}}\left(\operatorname{trace}\left(\Gamma_{c, p}\right)+\operatorname{trace}\left(\Phi_{c, p}\right)\right)\right\},
$$


Denote $\Gamma_{c, p}^{*}, \Phi_{c, p}^{*}, p=\overline{1, s 2^{m}}$ as an optimal solution of (83). At time $k$, for a given state $x(k)$, let $v^{*}(k)$ be the optimal solution of the following optimization problem,

$$
\begin{aligned}
& \min _{v(k)} \max _{q=\overline{1, s 2^{m}}}\left\{\frac{1}{2} x(k)^{T} \Gamma_{c, p}^{*} x(k)+\frac{1}{2} v(k)^{T} \Phi_{c, p}^{*} v(k)\right\}, \\
& \text { s.t. }\left[x(k)^{T} v(k)^{T}\right]^{T} \in \bigcap_{p=1}^{s 2^{m}} \mathcal{E}\left(P_{c, p}\right)
\end{aligned}
$$

As in Section II-D, (84) can be decomposed into $s 2^{m}$ parallel sub-problems. To solve each subproblems, we employ the method [19] in conjunction with the Newton-Raphson based technique in [21]. Note that the online computational burden is reduced if the same $\Phi_{c, p}^{*}$ can be used for a set of vertices. $\Gamma_{c, p}^{*}$ can be different for different vertices.

The control signal is computed as

$$
u(k)=\operatorname{sat}\left(K x(k)+(L-K) v^{*}(k)\right)
$$

Corollary 4: The controller (85) guarantees recursive feasibility and robust asymptotic stability for all feasible initial states.

Proof: It is omitted here.

In the following, the prediction dynamics based MPC with saturated feedback is summarized. It consists of two stages: offline stage and online stage.

\section{Offline Stage}

1: Select the matrix gain $L$.

2: Optimize $H, V$ by solving (69) for $H$, and by solving a corresponding problem for $V$.

3: Optimize $P_{c, p}, \forall p=\overline{1, s 2^{m}}$ by using Corollary 2.

4: Optimize $\Gamma_{c, p}^{*}, \Phi_{c, p}^{*}, \forall p=\overline{1, s 2^{m}}$ by solving (83).

\section{Online Stage}

1: Measure/estimate $x(k)$.

2: Obtain $v^{*}(k)$ by solving (84).

3: Apply the control law $u(k)=\operatorname{sat}\left(K x(k)+(L-K) v^{*}(k)\right)$ to (1).

\section{EXAMPLES}

Two example systems are shown in this section. The CVX toolbox [14] was used to solve the SDP optimization problems. For comparison purposes, the prediction dynamics based MPC 
methods with linear feedback when a common quadratic function [1], and a set of quadratic functions are used, are denoted as algorithm 1, and algorithm 2, respectively. The prediction dynamics based MPC method with saturated feedback is denoted as algorithm 3.

\section{A. Example 1}

To illustrate the concept of the intersection of ellipsoids used to characterize of the domain of attraction, a very simple example is presented. Consider the system (1) with $A_{1}=0.25, A_{2}=$ $1.5, B_{1}=1, B_{2}=2$. There are only input constraints $-1 \leq u \leq 1$.

The weighting matrices are $Q=1, R=0.01$. The unconstrained LQ controller is obtained by solving a SDP problem $K=-0.5833$. The gain $L$ is chosen as $L=-0.4268$. The auxiliary gain matrices are $V=-0.2570$, and $H=-0.2560$.

Fig. 1 presents the robustly invariant and constraint-admissible sets for algorithm $1, \mathcal{E}(P)$ (dash-dot yellow line), for algorithm $2, \mathcal{E}\left(P_{1}\right) \cap \mathcal{E}\left(P_{2}\right)$ (dashed red lines), and for algorithm 3, $\bigcap_{p=1}^{4} \mathcal{E}\left(P_{c, p}\right)$ (solid blue lines). It can be observed that $\mathcal{E}(P) \subset\left(\mathcal{E}\left(P_{1}\right) \cap \mathcal{E}\left(P_{2}\right)\right) \subset \bigcap_{p=1}^{4} \mathcal{E}\left(P_{c, p}\right)$. The ellipsoid matrices are

$$
\begin{gathered}
P=\left[\begin{array}{cc}
8.4881 & 14.6720 \\
14.6720 & 32.2985
\end{array}\right], P_{1}=\left[\begin{array}{cc}
20.3697 & 58.8904 \\
58.8904 & 196.8595
\end{array}\right], P_{2}=\left[\begin{array}{cc}
16.6790 & -4.5257 \\
-4.5257 & 77.2940
\end{array}\right], \\
P_{c, 1}=\left[\begin{array}{ll}
15.2260 & 11.2142 \\
11.2142 & 409.0984
\end{array}\right], P_{c, 2}=10^{6}\left[\begin{array}{cc}
0.4497 & 0.6441 \\
0.6441 & 2.3785
\end{array}\right], \\
P_{c, 3}=\left[\begin{array}{cc}
222.0988 & -146.1684 \\
-146.1684 & 214.7797
\end{array}\right], P_{c, 4}=\left[\begin{array}{ll}
15.6129 & 10.0605 \\
10.0605 & 99.2449
\end{array}\right]
\end{gathered}
$$

Note that $\mathcal{E}\left(P_{c, p}\right) \subset \mathcal{E}\left(P_{c, 2}\right), p=1,2,4$. Hence $\bigcap_{p=1}^{4} \mathcal{E}\left(P_{c, p}\right)=\mathcal{E}\left(P_{c, 1}\right) \cap \mathcal{E}\left(P_{c, 3}\right) \cap \mathcal{E}\left(P_{c, 4}\right)$.

\section{B. Example 2}

This example is a classical angular positioning system [5]. The system consists of a rotating antenna at the origin of the plane, driven by an electric motor, see Fig. 2. The control problem is to rotate the antenna by applying the input voltage to the motor so that it always points in the direction of a moving object in the plane. The motion of the antenna can be described by the discrete-time system (1) obtained from its continuous-time counterpart by discretizations with a 


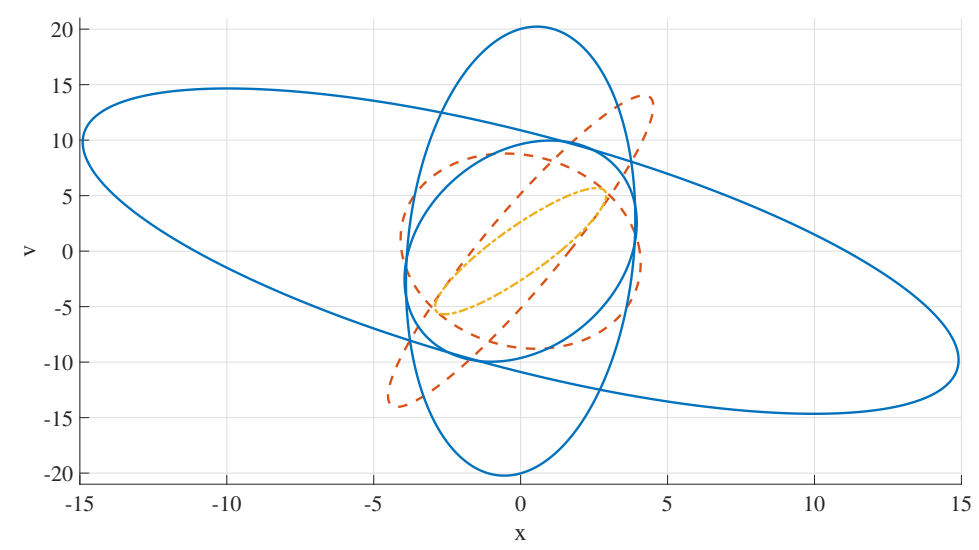

Fig. 1: Robustly invariant and constraint-admissible set for algorithm 1 (dash-dot yellow), for algorithm 2 (dashed red), for algorithm 3 (solid blue) for example 1.

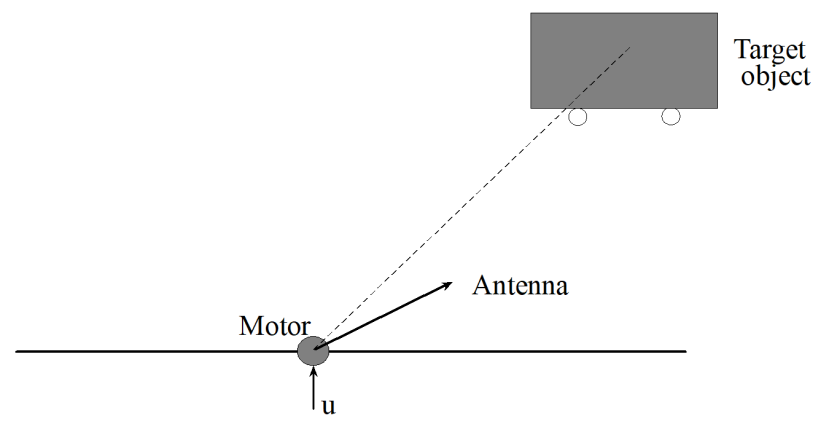

Fig. 2: Angular positioning system for example 2.

sampling time of $0.1 \mathrm{sec}$ and Euler's first-order approximation for the derivative. As the result, one obtains

$$
A_{1}=\left[\begin{array}{cc}
1 & 0.1 \\
0 & 1
\end{array}\right], A_{2}=\left[\begin{array}{cc}
1 & 0.1 \\
0 & 0
\end{array}\right], B_{1}=B_{2}=\left[\begin{array}{c}
0 \\
0.1574
\end{array}\right]
$$

The input and state constraints are: $-1 \leq u \leq 1,-3 \leq x_{1} \leq 3$. The weighting matrices are

$$
Q=\left[\begin{array}{ll}
1 & 0 \\
0 & 0
\end{array}\right], R=2 \times 10^{-5}
$$

The corresponding LQ gain matrix is $K=[-46.0650-7.7831]$. The gain matrix $L$ is chosen as $L=[-0.1479-1.4582]$. The auxiliary gain matrices are $V=\left[\begin{array}{ll}-10.0079-2.9145\end{array}\right]$, and 
$H=\left[\begin{array}{ll}-0.0307-0.0576\end{array}\right]$.

Fig. 3 shows the cut of the robustly invariant and constraint-admissible sets through $v=\mathbf{0}$ for algorithm 1 (dash-dot yellow line), for algorithm 2 (dashed red lines), and for algorithm 3 (solid blue line). Note that, since $v=\mathbf{0}$

- The prediction dynamics controller becomes $u(k)=K x(k)$ for algorithm 1 inside the dash-dot yellow set.

- The prediction dynamics controller becomes $u(k)=K x(k)$ for algorithm 2 inside the intersection of the two dashed red sets.

- The prediction dynamics controller becomes $u(k)=\operatorname{sat}(K x(k))$ for algorithm 3 inside the intersection of the four solid blue sets.

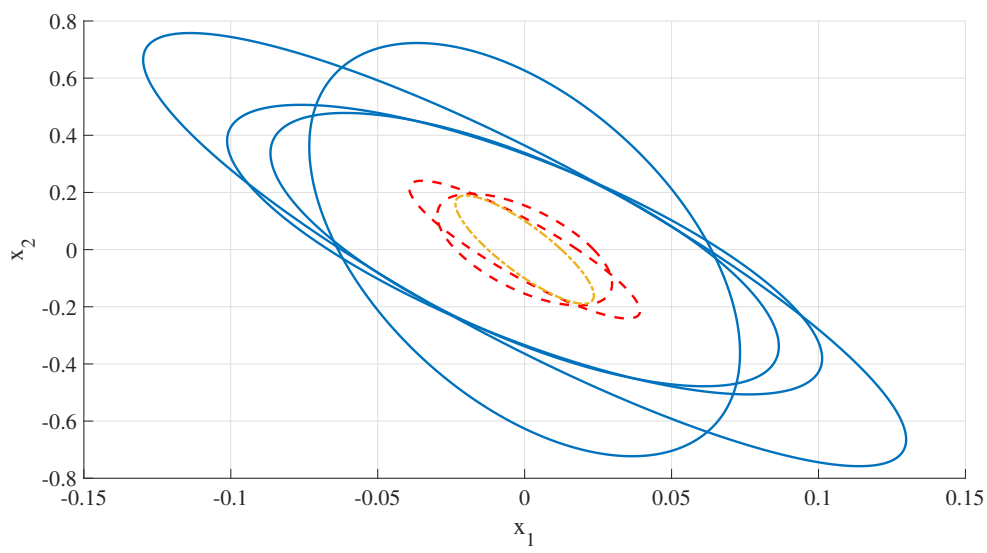

Fig. 3: Cut of the robustly invariant and constraint-admissible sets through $v=\mathbf{0}$ for algorithm 1 (dash-dot yellow), for algorithm 2 (dashed red), for algorithm 3 (solid blue) for example 2 .

For the initial condition $x(0)=\left[\begin{array}{ll}-1 & -0.5\end{array}\right]^{T}$, Fig. 4 shows the state and input trajectories of the closed-loop system as functions of time using algorithm 1 (dash-dot yellow), using algorithm 2 (dashed red), and using algorithm 3 (solid blue). Note that for algorithm 2, one matrix $\Phi$ is used to calculate the upper bound of the cost function. For algorithm 3, two matrices $\Phi_{c, 1}, \Phi_{c, 2}$ are used. Fig. 4(b) also presents the realization of $\lambda$.

Finally, using the TIC/TOC function of MATLAB 2020b, we found that the online computation times for one sampling interval were $1.2292 \times 10^{-4}[\mathrm{~s}], 2.9461 \times 10^{-4}[\mathrm{~s}]$, and $5.7352 \times 10^{-4}$ for algorithm 1 , for algorithm 2 , and for algorithm 3 , respectively. 

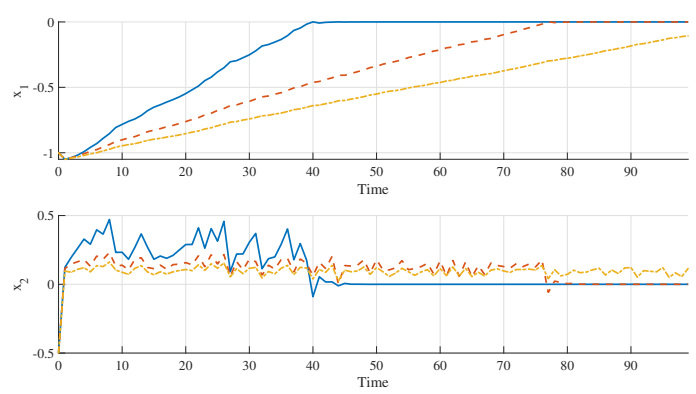

(a)
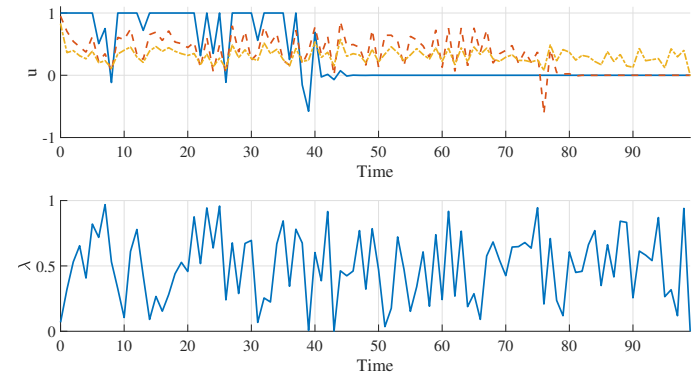

(b)

Fig. 4: (a) State, (b) Input and $\lambda$ realization trajectories as functions of time for algorithm 1 (dash-dot yellow), for algorithm 2 (dashed red), and for algorithm 3 (solid blue) for example 2.

\section{CONCLUSION}

Two novel prediction dynamics based MPC approaches are presented for constrained discretetime systems with uncertain and/or time-varying polytopic uncertainties. A particular choice of the parameters of the prediction dynamics with linear/saturated feedback is proposed without solving any optimization problem. This choice has the same desired property as that of earlier solutions in the literature, i.e., the domain of attraction of the controlled system under a linear/saturated dynamic feedback law is identical to the domain of attraction under any static linear/saturated state feedback law. To describe the domain of attraction as well as to estimate the upper bound of the cost function, the algorithms use a set of quadratic functions, each one corresponding to a different vertices of the uncertainty polytope and/or the saturated inputs. The design procedures do not require the assumption of quadratic stability. Two numerical examples with comparison to earlier solutions in the literature demonstrate the effectiveness of the new methods.

\section{REFERENCES}

[1] M. Cannon and B. Kouvaritakis, “Optimizing prediction dynamics for robust mpc," IEEE Transactions on Automatic Control, vol. 50, no. 11, pp. 1892-1897, 2005.

[2] H.-N. Nguyen, "Optimizing prediction dynamics with saturated inputs for robust model predictive control," IEEE Transactions on Automatic Control, vol. 66, no. 1, pp. 383-390, 2020.

[3] B. Kouvaritakis, M. Cannon, and J. A. Rossiter, "Who needs qp for linear mpc anyway?” Automatica, vol. 38, no. 5, pp. 879-884, 2002. 
[4] L. Imsland, N. Bar, and B. A. Foss, "More efficient predictive control,” Automatica, vol. 41, no. 8, pp. 1395-1403, 2005.

[5] M. V. Kothare, V. Balakrishnan, and M. Morari, "Robust constrained model predictive control using linear matrix inequalities," Automatica, vol. 32, no. 10, pp. 1361-1379, 1996.

[6] A. Bemporad, F. Borrelli, and M. Morari, "Min-max control of constrained uncertain discrete-time linear systems," IEEE Transactions on automatic control, vol. 48, no. 9, pp. 1600-1606, 2003.

[7] F. A. Cuzzola, J. C. Geromel, and M. Morari, "An improved approach for constrained robust model predictive control," Automatica, vol. 38, no. 7, pp. 1183-1189, 2002.

[8] T. Hu, Z. Lin, and B. M. Chen, "Analysis and design for discrete-time linear systems subject to actuator saturation," Systems \& control letters, vol. 45, no. 2, pp. 97-112, 2002.

[9] M. C. De Oliveira, J. Bernussou, and J. C. Geromel, “A new discrete-time robust stability condition," Systems \& control letters, vol. 37, no. 4, pp. 261-265, 1999.

[10] H.-N. Nguyen, Constrained Control of Uncertain, Time-Varying, Discrete-Time Systems: An Interpolation-Based Approach. Springer, 2013, vol. 451.

[11] F. Blanchini and S. Miani, Set-theoretic methods in control. Springer, 2008.

[12] T. Hu, A. R. Teel, and L. Zaccarian, "Stability and performance for saturated systems via quadratic and nonquadratic lyapunov functions," IEEE Transactions on Automatic Control, vol. 51, no. 11, pp. 1770-1786, 2006.

[13] R. Goebel, T. Hu, and A. R. Teel, "Dual matrix inequalities in stability and performance analysis of linear differential/difference inclusions," in Current trends in nonlinear systems and control. Springer, 2006, pp. 103-122.

[14] M. Grant, S. Boyd, and Y. Ye, "Cvx: Matlab software for disciplined convex programming," 2009.

[15] J. Lofberg, "Yalmip: A toolbox for modeling and optimization in matlab," in 2004 IEEE international conference on robotics and automation (IEEE Cat. No. 04CH37508). IEEE, 2004, pp. 284-289.

[16] M. Vidyasagar, Nonlinear systems analysis. SIAM, 2002.

[17] S. Boyd, S. P. Boyd, and L. Vandenberghe, Convex optimization. Cambridge university press, 2004.

[18] Y. Nesterov and A. Nemirovskii, Interior-point polynomial algorithms in convex programming. SIAM, 1994.

[19] S.-P. Han and G. Lou, "A parallel algorithm for a class of convex programs," SIAM Journal on Control and Optimization, vol. 26, no. 2, pp. 345-355, 1988.

[20] A. N. Iusem and A. R. De Pierro, "On the convergence of han's method for convex programming with quadratic objective," Mathematical Programming, vol. 52, no. 1, pp. 265-284, 1991.

[21] B. Kouvaritakis, J. A. Rossiter, and J. Schuurmans, "Efficient robust predictive control," IEEE Transactions on automatic control, vol. 45, no. 8, pp. 1545-1549, 2000.

[22] T. Alamo, A. Cepeda, and D. Limon, "Improved computation of ellipsoidal invariant sets for saturated control systems," in Proceedings of the 44th IEEE Conference on Decision and Control. IEEE, 2005, pp. 6216-6221. 